\title{
Comprendre les réactions au risque
}

\author{
Boudreau $\mathrm{JA}^{1 *}$
}

\author{
Affiliation \\ ${ }^{1}$ Institut national de la recherche scientifique, Centre Urbanisation Culture Société, Montréal (Québec) \\ *Correspondance : julie-anne.boudreau@ucs.inrs.ca
}

Citation proposée : Boudreau JA. Comprendre les réactions au risque. Relevé des maladies transmissibles au Canada 2016;42:233-4. https://doi.org/10.14745/ccdr.v42i10a08f

\section{Contexte}

Nous vivons à une époque marquée par une urbanisation intense - de plus en plus de gens vivent dans des villes qui s'agrandissent et occupent plus de territoire. L'urbanisation signifie également que les modes de vie urbains, en particulier les styles urbains de relation au temps, à l'espace et à l'affect, sont dominants $(1,2)$. La prise de risque et la peur sont de plus en plus répandues dans les milieux urbains, notamment chez les jeunes qui pratiquent des sports extrêmes, font de l'activisme "risqué ", consomment des drogues ou commettent des actes de délinquance. Bien que ces formes de prise de risque soient différentes de la décision de suivre ou non les directives de santé publique, nous pouvons néanmoins les utiliser pour comprendre comment, dans un monde urbain, nos relations au temps et à l'affect sont en train de changer en profondeur par rapport à ce que nous connaissions auparavant.

\section{Objectif}

À partir de travaux sur le terrain, l'objectif est de fournir une analyse sociologique des questions suivantes: Comment la peur influe-t-elle sur le comportement des gens au quotidien dans les villes? Comment pourrions-nous comprendre les personnes qui se mettent volontairement en danger? Que signifie la prévenance (care) dans les milieux urbains anonymes? Les réponses à ces questions contribueront à éclairer les communications de santé publique sur les risques émergents liés aux maladies à transmission vectorielle (MTV).

\section{Narratif}

Commençons par deux histoires. En avril 2009, la ville de Mexico était aux prises avec l'épidémie de grippe H1N1. Un vent de panique soufflait sur la ville. Des rumeurs reliaient diverses sources de peur (trafiquants de drogue, crise économique, corruption de l'État et tremblements de terre). D'un point de vue sociologique, ces rumeurs révélaient un manque de confiance dans les autorités publiques nationales et internationales. Au final, toutefois, le paysage quotidien habituel de la ville a été transformé lorsque les gens ont commencé à suivre les mesures préventives : porter des masques, ne plus se serrer la main. En mars 2016, on a craint une autre épidémie de grippe. Mais la panique ne s'est pas emparée de la ville. Au contraire, les gens savaient quoi faire et à quoi s'attendre. Ils ont porté des masques et adapté leurs comportements.
En même temps, un autre risque menaçait la ville : le pire épisode de smog en 14 ans. La cause? Entre 2005 et 2013, le nombre de voitures dans la ville a augmenté en flèche, passant de 3,5 à 6,8 millions. En outre, au cours de ces semaines, une stabilité atmosphérique sans précédent, des pressions élevées et un rayonnement solaire important empêchaient les polluants de quitter l'atmosphère. Le gouvernement a déclaré une urgence de phase 1, sur une échelle allant de la pré-urgence à la phase 2. Le système de transports en commun est devenu gratuit pour encourager les gens à laisser leur voiture à la maison. De plus, le gouvernement a décidé de doubler le nombre de voitures interdites à la circulation un jour donné (diminuant le nombre de voitures de deux millions). La réaction a été immédiatement négative.

La juxtaposition de ces deux exemples vise à démontrer ce qui suit. Lorsque les gens craignent une maladie immédiate (comme la grippe), ils obéissent aux mesures préventives. En revanche, lorsque le risque est plus éloigné ou à plus long terme (comme avoir un cancer dans le futur), ils peuvent refuser de s'y plier, car ils ne souhaitent pas abandonner leur confort $(3,4)$. Le caractère immédiat est la clé ici. Il est difficile d'encourager la population à penser aux conséquences pour l'avenir.

Il ne suffit pas d'un fondement biologique ou statistique pour que les risques soient établis dans l'esprit du public. Pour qu'un risque soit pris au sérieux par un groupe cible, il doit devenir un problème social. Pour qu'il prenne de l'importance dans la sphère publique, il doit être politiquement considéré comme un problème. Sinon, les gens ne prêteront pas attention au risque ou n'en seront même pas conscients. À mesure que nous évoluons vers un monde composé de villes, la conceptualisation du futur semble devenir de plus en plus difficile et, par conséquent, la motivation pour modifier le risque pourrait s'en trouver amoindrie.

Il est incorrect de supposer que les gens voudront répondre à un risque mesurable. Cette hypothèse ne tient pas compte du fait que, pour qu'un risque soit intelligible, il doit être considéré comme un problème public. Pour reprendre l'exemple de la crise de la grippe H1N1 à Mexico en 2009, la population ne faisait pas confiance aux institutions publiques, en raison d'un manque de cohérence entre la façon moderne, rationnelle, scientifique de poser le problème et la façon dont les gens vivaient et ressentaient leur vie urbaine au quotidien. Placer des problèmes au centre des préoccupations publiques est une affaire de politique. Le changement climatique, par exemple, n'était pas un problème " qui faisait jaser » il y a une dizaine d'années (5). Même si les scientifiques en parlaient depuis des décennies, il n'était pas accepté dans le débat public comme un problème de 
société. Il a fallu un travail politique considérable pour le rendre intelligible aux yeux des acteurs de l'État et du grand public.

Les systèmes de gestion du risque sont trop techniques et dépolitisés et sont donc inefficaces, car ils n'ont pas de sens pour les personnes à qui ils s'adressent. Par exemple, les programmes de prévention de la délinquance chez les jeunes sont entièrement fondés sur des facteurs de risque, des calculs actuariels et des mesures d'évaluation. Cette description du risque - en l'occurrence, le risque de commettre des infractions mineures ou de consommer des drogues - ignore l'humanité et l'expérience vécue de la personne qui est la cible des programmes de prévention. Dans ce système, les jeunes ne sont plus considérés comme des individus ayant des désirs, des humeurs et des choix. Au contraire, ils sont considérés comme des paquets de données dans des statistiques de probabilité (6). Cette approche ne prend pas en compte le point de vue des jeunes qui peuvent penser que la vie serait assez ennuyeuse sans risque et que la prise de risque apporte un certain plaisir (7). Par essence, elle déshumanise la personne qui est la cible des programmes de prévention.

Calculer les risques et concevoir les mesures de prévention en conséquence n'est peut-être pas un moyen efficace de s'attaquer au risque émergent associé aux MTV dans les villes. Selon les mots d'un travailleur de rue chargé de la prévention chez les jeunes à Montréal-Nord (18 novembre 2008) : “ C'est important d'humaniser le gang. Dans le gang, il y a des individus qui ont des vies brisées. " Une approche efficace doit reconnaître et tenir compte de l'expérience vécue des personnes et considérer politiquement le risque comme un problème pour qu'il devienne un risque socialement accepté. Nous devons humaniser notre approche et parler une langue que les personnes peuvent entendre : celle de la prévenance (care).

\section{Conclusion}

Pourquoi les gens continuent-ils à prendre des risques même si cela semble " irrationnel »? Deux éléments de réponse, étroitement liés, peuvent être apportés : parce qu'ils ne font pas confiance aux autorités de l'État (8) et parce qu'ils ne se sentent pas reconnus en tant qu'individus ayant chacun des désirs, des besoins et des sentiments uniques.

Les systèmes de gestion du risque ont malheureusement pour caractéristique de penser de façon systématique et non en termes d'interactions humaines $(7,9)$. S'il n'y a pas de confiance, si les personnes ne se sentent pas reconnues en tant qu'individus, alors le processus crucial de politisation d'un problème n'aura pas lieu. Dans ce cas de figure, le problème n'apparaîtra pas sur le radar social, et toutes les communications relatives au risque seront perçues comme sans importance.

La prévenance (care) a fondamentalement à voir avec les interactions humaines. Les gens $n$ 'isolent pas un seul risque (par exemple, les MTV), ils prennent des décisions fondées sur leur vie tout entière. Et nous ne pourrons le comprendre que si nous humanisons notre approche de la prévention du risque.

\section{Conflit d'intérêts}

Aucun.

\section{Références}

1. Boudreau JA. Global urban politics: Informalisation of the state. Cambridge: Polity Press; 2017.

2. Boudreau JA. Urbanity as a way of life: Risky behaviour, creativity, and post-heroism in Canada and Mexico. In: Vincent-Geslin S, Adly H, Pedrazzini Y, Zorro Y (éditeurs). Translating the city: Interdisciplinarity in urban studies. Lausanne et Oxford: EPFL Press Routledge; 2015.

3. Boudreau JA, Wood PK. (Dis)comfort, (in)security and the experience of the spaces in-between. In: Young D, Keil $\mathrm{R}$, Wood PK (éditeurs). In-between infrastructure: Urban connectivity in an age of vulnerability. Kelowna, BC: Praxis (e) Press; 2011. p. 209-23.

4. Boudreau JA, Liguori M, Séguin-Manegre M. Fear and youth citizenship practices: Insights from Montréal. In: Citizenship studies. 2015; doi:10.1080/13621025.2015.1006177.

5. Keil R, Boudreau JA. Metropolitics and metabolics: Rolling out environmentalism in Toronto. In: Swyngedouw E, Heynen N, Kaika M (éditeurs). Urban political ecology. London: Routledge; 2006. p. 41-62.

6. Boudreau JA. Jeunes et gangs de rue : I'informel comme lieu et forme d'action politique à Montréal. ACME : Revue électronique internationale de géographie critique. 2013;12(3):520-550.

7. Lupton D, Tulloch J. Life would be pretty dull without risk: Voluntary risk-taking and its pleasures. Health Risk Soc. 2002;4(2). doi:10.1080/13698570220137015.

8. Le Breton D. The anthropology of adolescent risktaking behaviours. Body Soc. 2004;10(1):1-15. doi:10.1177/1357034X04041758.

9. Lyng S. Edgework: A social psychological analysis of voluntary risk taking. AJS. 1990;95(4):851-886. doi:10.1086/229379. 